APJ LETTERS, IN PRESS

Preprint typeset using $\mathrm{LT}_{\mathrm{E}} \mathrm{X}$ style emulateapj v. 6/22/04

\title{
SUPERBURST IGNITION AND IMPLICATIONS FOR NEUTRON STAR INTERIORS
}

\author{
EDWARD F. BROWN \\ Department of Physics and Astronomy and the Joint Institute for Nuclear Astrophysics, Michigan State University, East Lansing, MI 48824 \\ Draft version September 27, 2018
}

\begin{abstract}
Superbursts are thought to be powered by the unstable ignition of a carbon-enriched layer formed from the burning of accreted hydrogen and helium. As shown by Cumming \& Bildsten, the short recurrence time hinges on the crust being sufficiently hot at densities $\rho>10^{9} \mathrm{~g} \mathrm{~cm}^{-3}$. In this Letter, we self-consistently solve for the flux coming from the deep crust and core. The temperature where the carbon unstably ignites is only weakly sensitive to the composition of the ashes of $\mathrm{H} / \mathrm{He}$ burning, but does depend on the thermal conductivity of the inner crust and the neutrino emissivity of the core. The observed superburst recurrence times and energetics suggest that the crust thermal conductivity is low, as if the crust were amorphous instead of crystalline. If the conductivity is higher, such as from a lattice with impurities, then matching the superburst properties require that the neutrino emissivity be not stronger than modified Urca. Observations of superbursts-energetics, recurrence times, and cooling times-therefore complement observations of isolated cooling neutron stars and soft X-ray transients in constraining properties of dense matter. Perhaps the most interesting object in this regard is KS 1731-260, which produced a superburst during its protracted accretion outburst but had a rapidly declining quiescent luminosity.

Subject headings: dense matter-nuclear reactions, nucleosynthesis, abundances—stars: neutron-X-rays: binaries-X-rays: bursts
\end{abstract}

\section{INTRODUCTION}

With the launch of BeppoSAX and RXTE, long-term monitoring of low-mass X-ray binaries became feasible. One fruit of this was the discovery of superbursts: these have a rapid rise and long decay, analogous to type I X-ray bursts, but are roughly a thousand times more energetic (Cornelisse et al. 2000; in't Zand et al. 2000; Kuulkers 2001; Kuulkers et al. 2002; Kuulkers 2002; Strohmaver \& Brown 2001; Wiinands 2001). The recurrence time is of order a year; for one source, 4U 1636-53, two superbursts were observed separated by $4.7 \mathrm{yr}$ (Wiinands 2001). Although most superbursts are observed at mass accretion rates $0.1-0.3 \dot{M}_{\text {Edd }}$, $\dot{M}_{\text {Edd }} \approx 10^{18} \mathrm{~g} \mathrm{~s}^{-1}$ being the Eddington accretion rate, recently in't Zand. Cornelisse. \& Cumming (2004) detected superbursts from the rapidly accreting source GX $17+2$.

Despite their similarities to type I X-ray bursts, the fuel for the superbursts was not immediately apparent. Taam \& Picklum (1978) had originally investigated the thermal stability of a pure ${ }^{12} \mathrm{C}$ layer and found that such a layer would unstably ignite when the accumulated mass was $\sim 10^{27} \mathrm{~g}$. This produces a very energetic burst, $E \sim$ $10^{44} \mathrm{ergs}$, with a recurrence time of $300 \mathrm{yr}\left(\dot{M} / 0.1 \dot{M}_{\text {Edd }}\right)$. Brown \& Bildsten (1998) demonstrated that carbon flashes with greatly reduced recurrence times could occur on a neutron star (NS) accreting pure He at a locally super-Eddington rate, such as might happen on an accreting X-ray pulsar. This explanation did not fit the observed superbursts because none of the sources showed pulsations in the persistent emission and, with the exception of $4 \mathrm{U} 1820-30$, all were thought to accrete a $\mathrm{H} / \mathrm{He}$ mixture that burned to matter with $A \sim 100$ (Schatz et al. 1999, 2001).

Cumming \& Bildsten (2001) suggested a resolution to this puzzle. They noted that only a small ${ }^{12} \mathrm{C}$ mass fraction $X\left({ }^{12} \mathrm{C}\right) \sim 0.1$ is sufficient to produce a thermonuclear runaway, and that the reduced conductivity of a heavy element

Electronic address: ebrown@pa.msu.edu mixture (e.g., 0.9:0.1 ${ }^{104} \mathrm{Ru}:{ }^{12} \mathrm{C}$ ) would produce a steeper thermal gradient for a fixed flux emergent from the deeper crust. The flux from the deep crust is powered by electron captures, neutron emissions and pycnonuclear reactions (Haensel \& Zdunik 1990, 2003) that release roughly 1.4 MeV per accreted nucleon; the outward-directed luminosity from the deep core is roughly $L_{\mathrm{nuc}} \lesssim 0.1 \mathrm{MeV}\left(\dot{M} / m_{\mathrm{u}}\right)$, for steadily accreting NSs with modified Urca neutrino cooling in their cores (Brown 2000). Here $m_{\mathrm{u}}$ is the mean nucleon mass. Under these conditions, superbursts would ignite for an accumulated mass $\sim 10^{25} \mathrm{~g}$, which is consistent with the observed energetics and recurrence times. The superbursts observed from GX $17+2$ are consistent with this scenario (in't Zand et al. 2004).

In this Letter, we calculate the thermal structure of the entire crust using a range of core neutrino emissivities and crust thermal conductivities. The flux in the carbon-enriched layer is not fixed, but increases with $\langle A\rangle /\left\langle Z^{2}\right\rangle$, where $\langle A\rangle$ and $\left\langle Z^{2}\right\rangle$ are the mean nuclear mass and squared nuclear charge of the carbon-enriched layer. As a result, the temperature at which superbursts ignite is relatively insensitive to the dominant isotope formed during $\mathrm{H} / \mathrm{He}$ burning. Furthermore, the temperature of this layer is sensitive to the nature of the thermal conductivity and neutrino emissivity of the deep crust and core. As a result, the superburst propertiesrecurrence times, energies, and cooling times-open a new view into the interior physics of NSs, complementary to observations of isolated cooling neutron stars (for a recent review, see Yakovlev \& Pethick 2004) and soft X-ray transients (Rutledge et al. 2001; Yakovlev et al. 2003; Yakovlev et al. 2004). In particular, the observed energetics and recurrence times agree with the scenario of Cumming \& Bildsten (2001) only if the nuclei in the NS crust are disordered or amorphous.

\section{THE THERMAL STRUCTURE OF THE CRUST}

To calculate the crust thermal structure, we use the method outlined in Brown (2000) with a few modifications. First, 
we take the core neutrino emissivity as a free parameter, rather than compute it self-consistently for a given equation of state (EOS). This is because our goal is to study the crust thermal structure for a given average core neutrino emissivity. To simplify our calculations, we follow the approach of Yakovlev \& Haensel (2003) and use an analytical density prescription $\rho=\rho_{\circ}\left[1-(r / R)^{2}\right]$ (Tolman 1939), where $\rho_{\circ}=15(8 \pi)^{-1} M R^{-3}$ with $R=\left(2 G M / c^{2}\right)\left[1-(1+z)^{-2}\right]^{-1}$ and $1+z=\left[1-2 G M /\left(R c^{2}\right)\right]^{-1 / 2}$. This prescription gives a reasonable approximation to the $\rho(r)$ found using modern nuclear EOS's (see Lattimer \& Prakash 2001, and references therein). With this choice for the EOS, the NS's mass $M$ and redshift $z$ specify its mechanical structure. We locate the crust-core boundary by a Maxwell construction using an EOS for the inner crust (Negele \& Vautherin 1973) and the core (Akmal. Pandharipande. \& Ravenhall 1998), which gives the transition density $\rho_{\mathrm{b}}=1.6 \times 10^{14} \mathrm{~g} \mathrm{~cm}^{-3}$.

With the core mass and radius specified, we compute the structure of the crust by integrating the general relativistic equation of hydrostatic balance from $P\left(\rho=\rho_{\mathrm{b}}\right)$ to $P=$ $g \times 10^{9} \mathrm{~g} \mathrm{~cm}^{-2}$. We then solve the thermal equations

$$
\begin{aligned}
e^{-v / 2} \partial_{r}\left(e^{v / 2} T\right) & =-(1+z) \frac{L}{4 \pi r^{2} K} \\
e^{-v} \partial_{r}\left(e^{v} L\right) & =4 \pi r^{2}(1+z)\left(\varepsilon_{\text {nuc }}-\varepsilon_{v}\right)
\end{aligned}
$$

by a relaxation method (Press et al. 1992). Here $T$ and $L$ denote proper values and $e^{v} \approx(1+z)^{-2}$. The condition at the crust/core boundary is $L\left(\rho=\rho_{b}\right)+L_{v}=0$ : the heat conducted into the core must be re-radiated as neutrinos. At a column depth $y=10^{9} \mathrm{~g} \mathrm{~cm}^{-2}$ we set $T=2.5 \times$ $10^{8} \mathrm{~K}$, in accordance with estimates for mixed $\mathrm{H} / \mathrm{He}$ ignition (Cumming \& Bildsten 2000).

The crust thermal profile is controlled by the EOS, the volumetric neutrino emissivity $\varepsilon_{v}$ and nuclear heating rate $\varepsilon_{\text {nuc }}$, and the thermal conductivity $K$. The crust EOS has contributions from degenerate, relativistic electrons, for which we use a tabulation of the Helmholtz free energy ${ }^{1}$ (Timmes \& Swesty 2000); from ions, for which we use the free-energy fits of Farouki \& Hamaguchi (1993); and from free neutrons, for which we use a compressible liquid-drop model (Mackie \& Bavm 1977). The neutrino emission in the inner crust is dominated by neutrino pair bremsstrahlung (Maxwell 1979), for which we use the rate calculated by Haensel. Kaminker. \& Yakovlev (1996). As in Brown (2000), we do not resolve the reaction layers in the deep crust, but instead use a distributed $\varepsilon_{\text {nuc }}$ normalized so that the total luminosity from the deep crust reactions is $L_{\mathrm{nuc}}=(1.4 \mathrm{MeV}) \times$ $\left(\dot{M} / m_{\mathrm{u}}\right)$.

Heat is conducted by electrons, and the thermal conductivity is given by the Wiedemann-Franz law, with the conductivity proportional to an electron-ion relaxation time $\tau$, for which we use the fitting formula of Potekhin et al. (1999), in the mean-ion approximation. A substantial uncertainty in computing the transport coefficients of the crust is its composition. During the lifetime of a low-mass binary, it is possible to replace a substantial portion of the $\sim 0.01 M_{\odot}$ crust. The accreted hydrogen and helium burn via the rp-process (Wallace \& Wooslev 1981); both steady-state (Schatz et al. 1999) and time-dependent, one-zone (Schatz et al. 2001) calculations predict that the ashes are a broad distribution

\footnotetext{
${ }^{1}$ Extension to nuclear densities courtesy F. Timmes
}

of ions peaking with $\langle A\rangle \approx 100$. Recent calculations of one-dimensional models of episodic accretion and unstable $\mathrm{H} / \mathrm{He}$ ignition for a range of accretion rates and metallicities find that repeated bursts drive the mean weight of the ashes to $\langle A\rangle \approx 64$ (Wooslev et al. 2004; Fisker et al. 2004). For definiteness, we use the compositional profile of Haensel \& Zdunik (2003), which presumes that the ashes of $\mathrm{H} /$ He burning have $\langle A\rangle \approx 100$; in the inner crust the composition is similar, however, to that of Haensel \& Zdunik (1990), which assumed the ashes of $\mathrm{H} / \mathrm{He}$ burning are ${ }^{56} \mathrm{Fe}$.

As pointed out by Cumming \& Bildsten (2001), the composition of the carbon-enriched layer can have a strong effect on the temperature profile. In this layer the ions are not crystallized and the conductivity scales roughly as $\langle A\rangle /\left\langle Z^{2}\right\rangle$. As a result, one would expect a steeper gradient for a heavier ion mixture and a given flux. The flux is not fixed, however, and must be determined self-consistently. Figure 1 shows the luminosity emergent from the crust for different values of $Z^{2} / A$ in the layer $10^{9} \mathrm{~g} \mathrm{~cm}^{-3}<y<10^{13} \mathrm{~g} \mathrm{~cm}^{-3}$. The connected squares mark the results of the calculations; the solid line indicates the slope $A / Z^{2}$. The luminosity is scaled by a fiducial value, $L_{\mathrm{C}}=1 \mathrm{MeV} \times\left(\dot{M} / m_{\mathrm{u}}\right)=9.6 \times 10^{34} \mathrm{ergs} \mathrm{s}^{-1} \dot{M}_{17}$, and the numbers beside each line indicate the accretion rate $\dot{M}_{17}=\dot{M} /\left(10^{17} \mathrm{~g} \mathrm{~s}^{-1}\right)$.

To understand why the thermal gradient depends weakly on the composition of the layer below the H/He burning shell, consider a simple two-zone model of the crust, with the heating from the pycnonuclear reactions at the interface between two zones. When most of the heat generated in the crust flows into the core, the maximum temperature in the crust, and therefore the thermal gradient between the H/He burning layer and the electron capture layers, is roughly fixed. This approximate constancy in the temperature gradient implies that the combination $L\left\langle Z^{2}\right\rangle /\langle A\rangle$ is roughly constant (Fig. 1). The superburst ignition temperature depends weakly on the composition of the carbon-enriched layer. At lower accretion rates and smaller $Z^{2} / A$, this simple argument weakens, as the luminosity in the outer layers becomes a larger fraction of $L_{\text {nuc }}$. For use in future computations, we fit the luminosity in the carbon-enriched layer as a function of $\dot{M}_{17}$ and $Z^{2} / A$ for $1<\dot{M}_{17}<10$ and $8<Z^{2} / A<20$. We write $L=q \dot{M} N_{\mathrm{A}}(1 \mathrm{MeV})=\left(9.65 \times 10^{34} \mathrm{ergs} \mathrm{s}^{-1}\right) \times q \dot{M}_{17}$, with

$$
q=0.386\left(\frac{Z^{2} / A}{12}\right)^{-0.6}\left(1-0.562 \dot{M}_{17}^{0.15}\right)
$$

This fit has an rms error of $1.7 \%$, with a maximum error of $5.1 \%$ for $\dot{M}_{17}=10$ and $Z^{2} / A=20$.

\section{SENSITIVITY TO THE PHYSICS OF THE DEEP CRUST AND CORE}

To explore the effects of the core and crust microphysics on the carbon ignition, we choose a core model with $M=$ $1.6 M_{\odot}$ and $z=0.35$, which implies that $R=10.5 \mathrm{~km}$ and $\rho_{\circ}=6.2 \rho_{\text {nuc }}=1.7 \times 10^{15} \mathrm{~g} \mathrm{~cm}^{-3}$. Here $\rho_{\text {nuc }} / m_{\mathrm{u}}=$ $0.16 \mathrm{fm}^{-3}$ is the fiducial nuclear density. Again following Yakovlev \& Haensel (2003), we use two cases of the core neutrino emissivity. The first case is $\rho \varepsilon_{v}=3.0 \times$ $10^{19} \mathrm{ergs} \mathrm{s}^{-1} \mathrm{~cm}^{-3}\left(T / 10^{9} \mathrm{~K}\right)^{8}$ throughout the core. This corresponds to a modified Urca process somewhat suppressed by superfluidity. For the second case, we add an enhanced emissivity $\rho \varepsilon_{V}=10^{26} \mathrm{ergs} \mathrm{s}^{-1} \mathrm{~cm}^{-3}\left(T / 10^{9} \mathrm{~K}\right)^{6}$ for $\rho>5.0 \rho_{\text {nuc }}$. This models the operation of an enhanced neutrino emissivity from, e.g., a direct Urca process above a density thresh- 


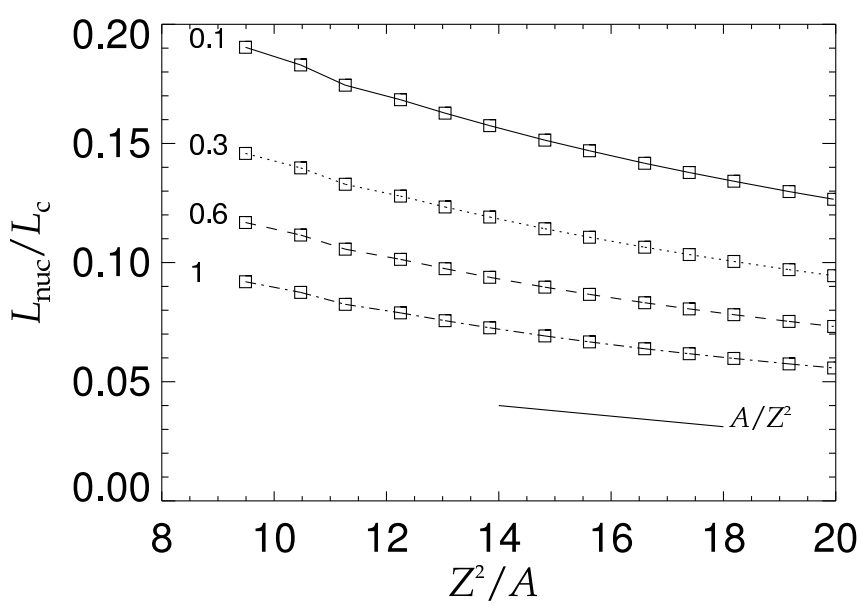

FIG. 1. - The emergent luminosity from the deep crust, scaled to $L_{\mathrm{C}}=$ $1 \mathrm{MeV} \times\left(\dot{M} / m_{\mathrm{u}}\right)$ for $\dot{M}_{17}=0.1,0.3,0.6$, and 10.0 (see text). This is for a crust with low thermal conductivity.

old (Lattimer et al. 1991). These choices change the core temperature by an order of magnitude for a given accretion rate: the proper temperature at the base of the crust is $T_{\mathrm{b}} \approx 4.6 \times 10^{8} \mathrm{~K}\left(\dot{M} / \dot{M}_{\text {Edd }}\right)^{1 / 8}$ for the first case and $T_{\mathrm{b}} \approx$ $3.9 \times 10^{7} \mathrm{~K}\left(\dot{M} / \dot{M}_{\text {Edd }}\right)^{1 / 6}$ for the second. The core temperature is insensitive to the exact value of the density threshold.

In addition to the neutrino emissivity of the core, we also choose two cases for the crust thermal conductivity. Calculations of the ionic state of the crust usually presume a single-species lattice, with possible impurities. The actual crust is formed, however, from the processing of rp-process ashes, and it is not known if the ions form an ordered lattice. We therefore pick two cases for comparison. First, that the lattice is disordered; we estimate the electron-ion relaxation time $\tau$ by setting the structure factor to unity (see Itoh \& Kohyama 1993). In this case $\tau^{-1}$ is independent of $T$ and proportional to $Z^{2} / A$. For the second case, we compute $\tau^{-1}=\tau_{\mathrm{e}, \mathrm{ph}}^{-1}+\tau_{\mathrm{e}, \mathrm{imp}}^{-1}$, where $\tau_{\mathrm{e}, \mathrm{ph}}^{-1}$ is the frequency of electronphonon scattering and $\tau_{\text {e.imp }}^{-1}$ is the frequency of electronimpurity scattering (Potekhin et al. 1999), for which we set $Q=\left\langle Z^{2}-\langle Z\rangle^{2}\right\rangle=100$. This is typical of the rp-process ashes, but it is larger than that of matter burned in a superburst (Schatz, Bildsten, \& Cumming 2003) or formed at the birth of the neutron star (Jones 2004).

The thermal profiles for these four cases are shown in Fig. 2 for $\dot{M}=3 \times 10^{17} \mathrm{~g} \mathrm{~s}^{-1}$ (approximately $\frac{1}{3}$ Eddington) and Fig. 3 for $\dot{M}=10^{18} \mathrm{~g} \mathrm{~s}^{-1}$ (approximately Eddington). The solutions with the higher core temperatures are for modified Urca processes; the ones with lower core temperatures for direct Urca processes. For each core temperature there are two profiles, one for a disordered, amorphous crust (solid lines) and one for an impure lattice (dotted lines).

The squares mark the point at which we estimate unstable ignition to occur. To define ignition, we use the criteria from Cumming \& Bildsten (2001) and set $\varepsilon_{1212}=$ $\xi \rho K T / y^{2}$, where $\varepsilon_{12,12}$ is the mass-specific heating rate from the reaction ${ }^{12} \mathrm{C}+{ }^{12} \mathrm{C}, \varepsilon_{\text {cool }}=\rho K T / y^{2}$ is an estimate of the cooling rate, and $\xi \approx 2 / 26$ is the ratio $\left(\partial_{T} \ln \varepsilon_{\text {cool }}\right) /\left(\partial_{T} \ln \varepsilon_{12,12}\right)$. This simple approach gives us a rough estimate of where ignition occurs and is known for $\mathrm{X}$-ray bursts to give results that are roughly in line with nu- merical simulations (Wooslev et al. 2004) and linear stability analyses (Naravan \& Hevl 2003). For the ${ }^{12} \mathrm{C}+{ }^{12} \mathrm{C}$ rate, we use the formula of Caughlan \& Fowler (1988) and incorporate strong screening according to Ogata. Ichimaru. \& van Horn (1993).

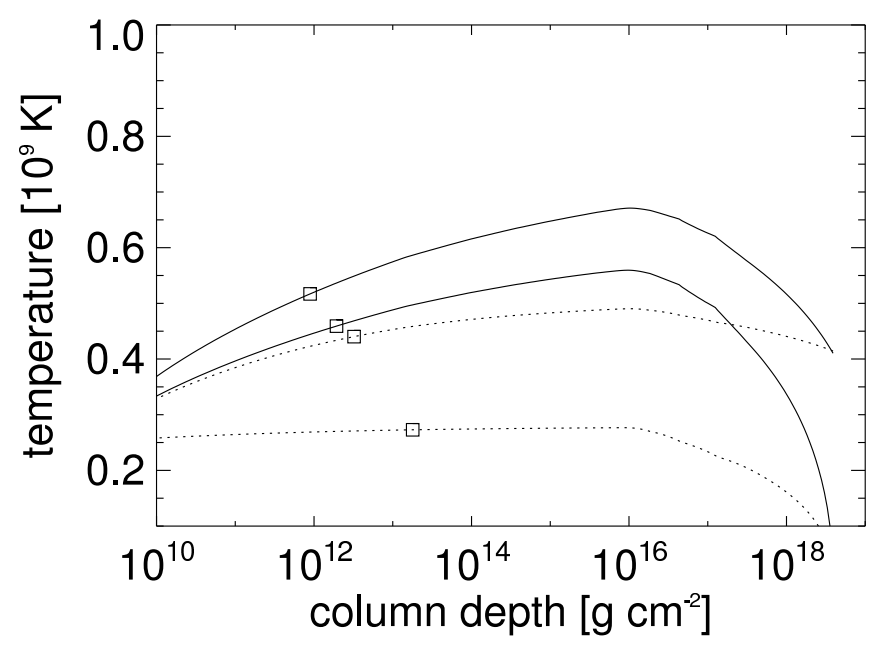

FIG. 2.-Crust temperatures for $\dot{M}=3.0 \times 10^{17} \mathrm{~g} \mathrm{~s}^{-1}$. The squares indicate where a mixture with $X\left({ }^{12} \mathrm{C}\right)=0.1$ would unstably ignite. The solid lines are for the crust thermal conductivity dominated by scattering; the dotted lines indicate a conductivity that is a mix of phonon and impurity scattering. The solutions with higher core temperatures correspond include only modified Urca processes; the solutions with a cold core temperature include in addition an enhanced neutrino emission.

For $\dot{M}=3.0 \times 10^{17} \mathrm{~g} \mathrm{~s}^{-1}$, the recurrence times for ${ }^{12} \mathrm{C}+{ }^{12} \mathrm{C}$ ignition are, from the hottest profile to the coldest, 1.4, 3.0, 5.1 , and 28 yr with total burst energies of $7.5 \times 10^{41}, 1.6 \times$ $10^{42}, 2.7 \times 10^{42}$ and $1.5 \times 10^{43} \mathrm{ergs} \mathrm{s}^{-1}$, respectively. Likewise, for $\dot{M}=10^{18} \mathrm{~g} \mathrm{~s}^{-1}$, the recurrence times are $0.08,0.10$, 0.30 , and $1.4 \mathrm{yr}$ and the total burst energies are $1.5 \times 10^{41}$, $1.7 \times 10^{41}, 5.3 \times 10^{41}$, and $2.5 \times 10^{42} \mathrm{ergs} \mathrm{s}^{-1}$. Neutron stars with enhanced neutrino cooling are not compatible with observed superburst recurrence times and energetics unless the crust is amorphous. Note that if the inner crust were to form a lattice with a low impurity concentration, $Q \sim 1$, then the ${ }^{12} \mathrm{C}+{ }^{12} \mathrm{C}$ ignition approaches the case discussed by Taam \& Picklum (1978), with recurrence times of 10-100 yr. For the case of a disordered lattice (solid curves), the ignition conditions become nearly independent of the core temperature at higher accretion rates.

\section{CONCLUSIONS}

Using parameterized models of the NS core, we have computed ignition conditions for ${ }^{12} \mathrm{C}$ that solve for the flux emergent from the inner crust. There are two principal conclusions. First, the flux in the carbon-enriched layer scales roughly as $\langle A\rangle /\left\langle Z^{2}\right\rangle$, so that the superburst ignition is only weakly dependent on the composition of that layer. Second, we confirm the scenario of Cumming \& Bildsten (2001), but only if the crust thermal conductivity is low. In particular, the calculated recurrence times are too long if there is both enhanced core neutrino emission and the crust thermal conductivity is set by phonon and impurity scattering. This is relevant not only to studies of neutron star cooling, but also to studies of the magnetic field evolution of the crust (for a recent study, see Cumming, Arras, \& Zweibel 2004).

In addition to the recurrence time and burst energetics, the cooling of the NS atmosphere, as evidenced by the 


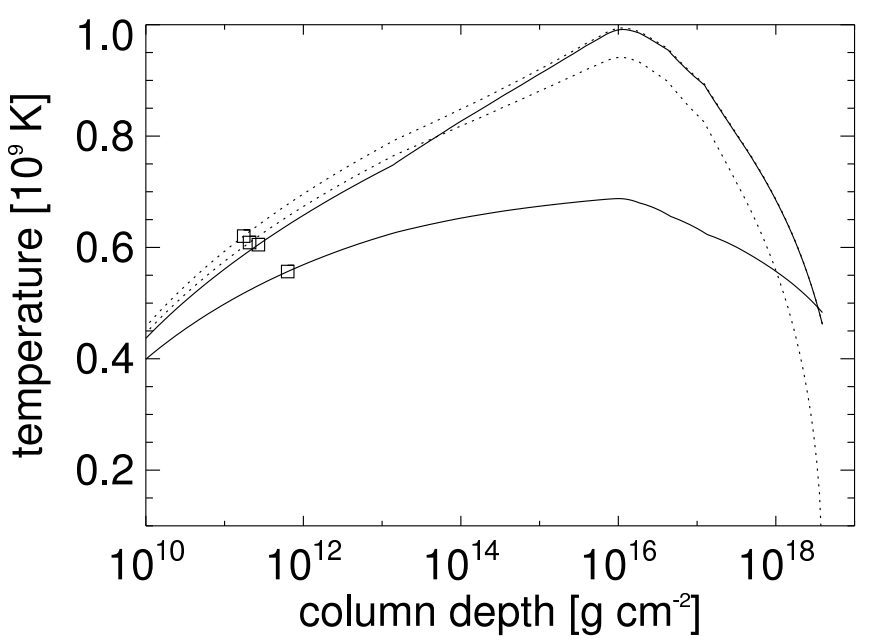

FIG. 3. - Same as but for $\dot{M}=10^{18} \mathrm{~g} \mathrm{~s}^{-1}$.

quenching of subsequent type I X-ray bursts, can be used to infer $y_{\text {ign }}$, the column depth of the superburst ignition (Cumming \& Macbeth 2004). The ignition curve, as estimated in $\S 3$ gives a unique temperature $T_{\mathrm{ign}}\left(y_{\mathrm{ign}}\right)$. Using this information thus reduces the uncertainty from the poorly constrained mass accretion rate. From Fig. 2 ign differs by a factor of 3-4 between different crust models for a core with only modified Urca processes.
Perhaps the most intriguing superburst source is KS 1731-260 (Kuulkers et al. 2002), for which there are independent estimates of the interior temperature and crust thermal conductivity. This source was observed to be persistent for $\approx 12 \mathrm{yr}$ until it suddenly went into quiescence (Wiinands et al. 2001). Rutledge et al. (2001) noted that because of its long outburst, the crust would be driven out of thermal equilibrium with the core, and therefore follow-up observations of the quiescent X-ray luminosity would measure the thermal relaxation of the crust. Such observations (Wiinands et al. 2002) revealed a rapid and strong decline of the X-ray luminosity. If the quiescent emission is solely powered by the cooling crust, this sharp decrease in the quiescent luminosity suggests that the crust has a high thermal conductivity, consistent with being a locally pure lattice, and that the neutrino emission from the core is stronger than modified Urca. The calculations in this paper, being steady-state, are not directly applicable to KS 1731-260, but do indicate that the low quiescent emission, combined with the occurrence of a superburst, are a challenge to our understanding of accreting neutron stars.

We thank Andrew Cumming, Bob Rutledge, Hendrik Schatz, and Alexander Heger for helpful conversations and comments.

\section{REFERENCES}

Akmal, A., Pandharipande, V. R., \& Ravenhall, D. G. 1998, Phys. Rev. C, 58 1804

Brown, E. F. 2000, ApJ, 531, 988

Brown, E. F. \& Bildsten, L. 1998, ApJ, 496, 915

Caughlan, G. R. \& Fowler, W. A. 1988, At. Data Nucl. Data Tables, 40, 283

Cornelisse, R., Heise, J., Kuulkers, E., Verbunt, F., \& in't Zand, J. J. M. 2000, ApJ, 357, L21

Cumming, A., Arras, P., \& Zweibel, E. 2004, ApJ, 609, 999

Cumming, A. \& Bildsten, L. 2000, ApJ, 544, 453

Cumming, A. \& Bildsten, L. 2001, ApJ, 559, L127

Cumming, A. \& Macbeth, J. 2004, ApJ, 603, L37

Farouki, R. \& Hamaguchi, S. 1993, Phys. Rev. E, 47, 4330

Fisker, J. L., Brown, E., Liebendoerfer, M., Schatz, H., \& Thielemann, F. K.

2004, Nucl. Phys. A, submitted, as part of proceedings, Nuclei in the Cosmos 8, astro-ph/0407319

Haensel, P., Kaminker, A. D., \& Yakovlev, D. G. 1996, A\&A, 314, 328

Haensel, P. \& Zdunik, J. L. 1990, A\&A, 227, 431

-. 2003, A\&A, 404, L33

in't Zand, J. J. M., Cornelisse, R., \& Cumming, A. 2004, A\&A, in press (astro-ph/0407083,

in't Zand, J. J. M., Kuulkers, E., Bazzano, A., Cornelisse, R., Cocchi, M. Heise, J., Muller, J. M., Natalucci, L., Smith, M. J. S., \& Ubertini, P. 2000, A\&A, 357, 520

Itoh, N. \& Kohyama, Y. 1993, ApJ, 404, 268

Jones, P. B. 2004, MNRAS, 351, 956

Kuulkers, E. 2001, The Astronomer's Telegram, 68, 1

-. 2002, A\&A, 383, L5

Kuulkers, E., in't Zand, J. J. M., van Kerkwijk, M. H., Cornelisse, R., Smith,

D. A., Heise, J., Bazzano, A., Cocchi, M., Natalucci, L., \& Ubertini, P. 2002, A\&A, 382, 503

Lattimer, J. M., Pethick, C. J., Prakash, M., \& Haensel, P. 1991, Phys. Rev. Lett., 66, 2701

Lattimer, J. M. \& Prakash, M. 2001, ApJ, 550, 426

Mackie, F. D. \& Baym, G. 1977, Nucl. Phys. A, 285, 332

Maxwell, O. V. 1979, ApJ, 231, 201
Narayan, R. \& Heyl, J. S. 2003, ApJ, 599, 419

Negele, J. W. \& Vautherin, D. 1973, Nucl. Phys. A, 207, 298

Ogata, S., Ichimaru, S., \& van Horn, H. M. 1993, ApJ, 417, 265

Potekhin, A. Y., Baiko, D. A., Haensel, P., \& Yakovlev, D. G. 1999, A\&A, 346,345

Press, W. H., Teukolsky, S. A., Vetterling, W. T., \& Flannery, B. P. 1992, Numerical Recipes in FORTRAN (Cambridge: Cambridge Univerisity Press)

Rutledge, R. E., Bildsten, L., Brown, E. F., Pavlov, G. G., Zavlin, V. E., \& Ushomirsky, G. 2001, ApJ, 580, 413

Schatz, H., Aprahamian, A., Barnard, V., Bildsten, L., Cumming, A., Ouellette, M., Rauscher, T., Thielemann, F.-K., \& Wiescher, M. 2001, Phys. Rev. Lett., 86, 3471

Schatz, H., Bildsten, L., \& Cumming, A. 2003, ApJ, 583, L87

Schatz, H., Bildsten, L., Cumming, A., \& Wiescher, M. 1999, ApJ, 524, 1014

Strohmayer, T. E. \& Brown, E. F. 2001, ApJ, 566, 1045

Taam, R. E. \& Picklum, R. E. 1978, ApJ, 224, 210

Timmes, F. X. \& Swesty, F. D. 2000, ApJS, 126, 501

Tolman, R. C. 1939, Physical Review, 55, 364

Wallace, R. K. \& Woosley, S. E. 1981, ApJS, 45, 389

Wijnands, R. 2001, ApJ, 554, L59

Wijnands, R., Guainazzi, M., van der Klis, M., \& Méndez, M. 2002, ApJ, 573, L45

Wijnands, R., Miller, J. M., Groot, P. J., Markwardt, C., Lewin, W. H. G., \& van der Klis, M. 2001, ApJ, 560, L159

Woosley, S. E., Heger, A., Cumming, A., Hoffman, R. D., Pruet, J., Rauscher, T., Fisker, J. L., Schatz, H., Brown, B. A., \& Wiescher, M. 2004, ApJS, 151,75

Yakovlev, D. G. \& Haensel, P. 2003, A\&A, 407, 259

Yakovlev, D. G., Levenfish, K. P., \& Haensel, P. 2003, A\&A, 407, 265

Yakovlev, D. G., Levenfish, K. P., Potekhin, A. Y., Gnedin, O. Y., \& Chabrier, G. 2004, A\&A, 417, 169

Yakovlev, D. G. \& Pethick, C. J. 2004, ARA\&A, in press, astro-ph/0402143 